\title{
Primeras observaciones de gaviotas patiama- rillas Larus michahellis Naumann, 1840 de origen cantábrico en la cuenca del río Ebro
}

\section{First records of Cantabrian yellow-legged gull Larus michahellis Naumann, 1840 within the Ebro river basin}

Juan Arizaga ${ }^{1 *}$, Alfredo Herrero ${ }^{1}$, Asier Aldalur ${ }^{1}$, Juan F. Cuadrado ${ }^{1}$

$\sigma^{*}$

\section{Resumen}

La dispersión de gaviotas patiamarillas Larus michahellis Naumann, 1840 de origen cantábrico al Mediterráneo es un fenómeno ya descrito. Se desconoce, no obstante, cuál es la ruta empleada en este desplazamiento. En este artículo se describe por primera vez la presencia de gaviotas patiamarillas de origen cantábrico en la cuenca del río Ebro y se especula, en consecuencia, sobre si el flujo de aves entre el Cantábrico y el Mediterráneo se da a través del Valle del Ebro. De un total de 13.132 avistamientos, relativos a 1.559 individuos diferentes marcados en colonias de Gipuzkoa, 2 aves (0,12\%) fueron observadas en diferentes puntos del Valle del Ebro, concretamente un ejemplar en el Vertedero de Culebrete (Tudela) y otro en el de Huesca, ambos en enero de 2015. Aunque el avistamiento de gaviotas cantábricas en la cuenca del río Ebro es escaso, la ausencia de observaciones tanto al norte de los Pirineos como a lo largo de la costa del sur de España podría apoyar la hipótesis de que el movimiento de gaviotas entre la costa cantábrica y el Mediterráneo sucede a través del Valle del Ebro.

Palabras clave: anillamiento, corredor migratorio, Gipuzkoa, Mediterráneo, movimientos, subadultos.

\section{Abstract}

The dispersal of Cantabrian yellow-legged gulls Larus michahellis Naumann, 1840 to the Mediterranean has been reported in previous works. It remains unknown, however, which route

\footnotetext{
1 Sociedad de Ciencias Aranzadi. Departmento de Ornitología

Zorroagagaina 11 • E-20014 Donostia-S. Sebastián, Spain

* Correspondencia: jarizaga@aranzadi.eus
} 
these birds may employ. In this work, the presence of yellow-legged gulls of Cantabrian origin in the Ebro river basin is described for the first time. This fact is considered to speculate on the possibility that yellow-legged gulls may follow the Ebro valley to fly between the Mediterranean sea and the Bay of Biscay. Out of 13.132 sightings, relating to 1.559 individual gulls ringed in a number of breeding colonies in Gipuzkoa, 2 of them (0.12\%) were seen in the Ebro valley; particularly, one bird was seen in the Culebrete landfill (Tudela), and another one was observed in a landfill in Huesca, both in January of 2015. Although the presence of Cantabrian yellowlegged gulls in the Ebro valley is marginal, the absence of sightings to the north of the Pyrenees and along the coast of southern Iberia suggests that movement from the Bay of Biscay to the Mediterranean may occur mainly along the Ebro river basin.

Key words: ringing, migratory flyway, Gipuzkoa, Mediterranean, movements, subadults.

\section{Laburpena}

Kaio hankahorien Larus michahellis Naumann, 1840 jatorri kantauriarrekoen Mediterraneoranzko dispertsioa jada deskribatuta dagoen gertakaria da. Mugimendu horretarako erabiltzen duten ibilbidea, ordea, ezezaguna da oraindik. Artikulu honetan, lehen aldiz, Ebro Haranean aurkitu diren jatorri kantauriarreko kaio hankahorien presentzia azaltzen da; era berean, hausnarketa bat egiten da ea hegazti horien Kantauri eta Mediterraneo arteko lekualdaketa Ebro Haranean zehar egiten oten duten.

Gipuzkoako kolonietan markatutako 1.559 aleri dagozkien 12.132 begiztatze datuen artean, bi ale $(\% 0,12)$ Ebro Haraneko puntu desberdinetan ikusi ziren; zehazki, ale bat Culebrete zabortegian (Tutera) eta beste bat Huescan, biak 2015eko urtarrilean. Nahiz eta Ebro Haranean jatorri kantauriarreko kaio gutxi begiztatu diren, Pirinioetan zein Espainia hegoaldeko kostan begiztatzerik ez egoteak hipotesi hau babes lezake: kaioek Kantauri-Mediterraneo artean mugitzeko Ebro Harana erabiltzen dutela.

Gako hitzak: eraztunketa, migrazio-korridorea, Gipuzkoa, Mediterraneoa, mugimenduak, gazteak.

La gaviota patiamarilla Larus michahellis Naumann, 1840 es un especie que se distribuye en las costas de España y Portugal, Mediterráneo occidental, noroeste de África y Macaronesia (Olsen \& Larson, 2004). Recientemente, ha ampliado su área de distribución hacia el norte, ocupando algunas zonas húmedas de Centroeuropa y la costa oeste de Francia (Geroudet, 1984; Yésou, 1991). Igualmente, también se observa como reproductora en el interior de países de la región mediterránea, como España (Bermejo \& Mouriño, 2003).

En la península ibérica nidifican dos de las tres subespecies que se han llegado a describir (Olsen \& Larson, 2004): L. m. michahellis, a lo largo de la costa mediterránea y en el interior, y L. m. lusitanius, desde la costa vasca hasta el centro de Portugal (sur de Portugal según Pons et al., 2004). Aunque L. m. Iusitanius es sedentaria (Munilla, 1997; Arizaga et al., 2010), 
la dispersión de individuos subadultos hacia la región mediterránea es un fenómeno ya descrito, sobre todo entre las poblaciones más orientales (Arizaga et al., 2009; Arizaga et al., 2010). Se ha especulado hasta cierto punto sobre si este movimiento CantábricoMediterráneo (o Mediterráneo-Cantábrico) tiene lugar a través del Valle del Ebro o, alternativamente, a través de los cauces que se extienden a lo ancho del norte de los Pirineos o incluso a través de una ruta que bordearía la península ibérica (Martínez-Abrain et al., 2002; Arizaga et al., 2009).

Desde que en 2005 se iniciara en Gipuzkoa un proyecto de anillamiento de gaviotas patiamarillas mediante marcas visuales (anilla de lectura a distancia), son miles los avistamientos que se han obtenido. El objetivo de este artículo es compilar los avistamientos del conjunto de aves marcadas en Gipuzkoa y analizar su dispersión, haciendo especial hincapié en las rutas que hayan podido seguir para llegar a las zonas donde son observadas. Especial atención se prestará a la presencia de la especie en la cuenca del río Ebro. En este contexto se comparan los avistamientos en la cuenca del Ebro con el total de avistamientos de gaviotas patiamarillas marcadas en Gipuzkoa realizados hasta la fecha.

Durante el periodo 2005-2014, un total de 2599 pollos y 63 adultos de gaviota patiamarilla fueron anillados con anillas de PVC de lectura a distancia en cuatro colonias de la provincia de Gipuzkoa (Fig. 1): Ulia, Santa Clara, Mollarri y Guetaria (Tabla 1).

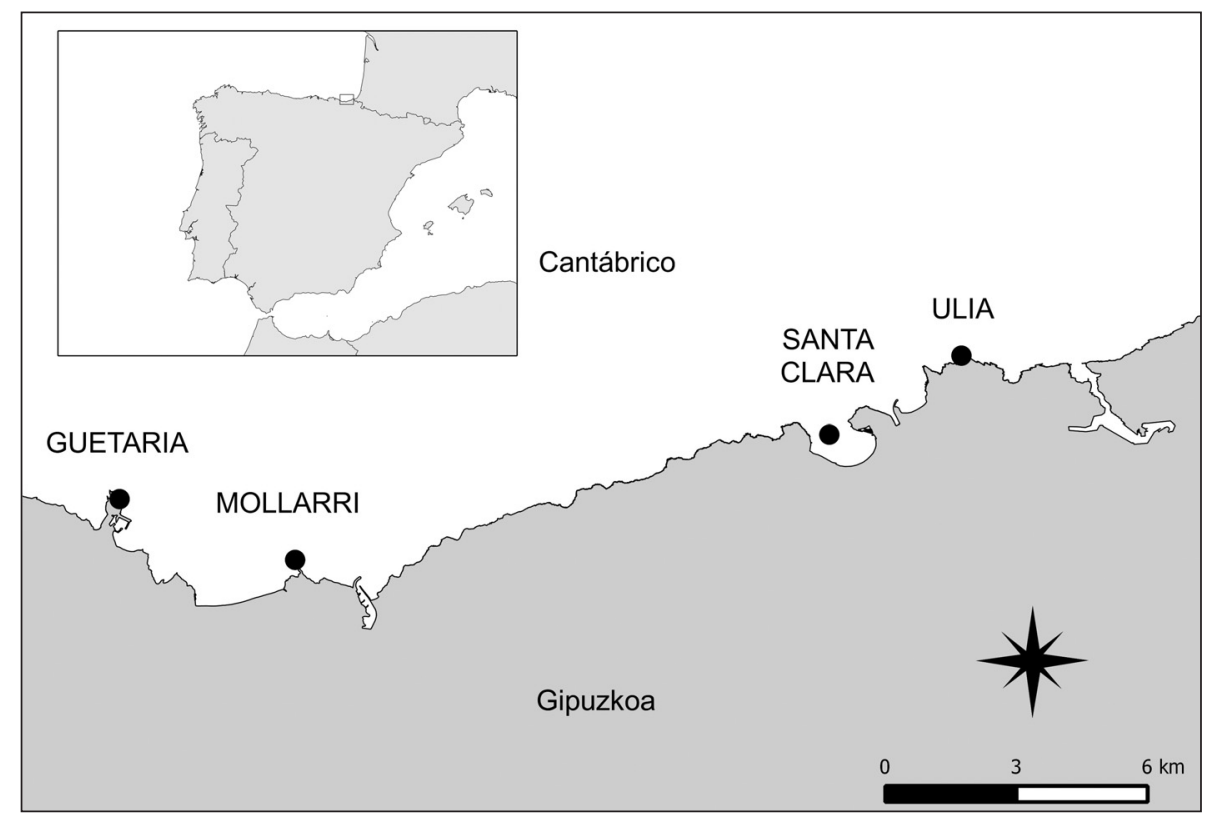

Fig. 1.- Localización en Gipuzkoa de las colonias donde se llevó a cabo el anillamiento de 2.599 pollos y 63 adultos de gaviota patiamarilla durante el periodo 2005-2014.

Fig. 1.- Location in Gipuzkoa of the four breeding colonies where a total of 2.599 yellow-legged gull chicks, and 63 adults were ringed during the period 2005-2014. 


\begin{tabular}{|rrrcr|}
\hline Año & Ulia & Santa Clara & Mollarri & Guetaria \\
\hline 2005 & 17 & 23 & 13 & 0 \\
2006 & 147 & 69 & 0 & 30 \\
2007 & $202(10)$ & 85 & 12 & 10 \\
2008 & $208(20)$ & 55 & 8 & 38 \\
2009 & $263(22)$ & 50 & 0 & 20 \\
2010 & $221(10)$ & 42 & 0 & 59 \\
2011 & 200 & 37 & 0 & 32 \\
2012 & 178 & 87 & 0 & 61 \\
2013 & 68 & 54 & 0 & 50 \\
2014 & 151 & 59 & 0 & $50(1)$ \\
\hline Total & $1655(62)$ & 561 & 33 & $350(1)$ \\
\hline
\end{tabular}

Tabla 1.- Número de pollos y adultos de gaviota patiamarilla anillados con anilla de PVC durante el periodo 2005-2014 en cuatro colonias de Gipuzkoa. El número de adultos se indica entre paréntesis.

Table 1.- The number of yellow-legged gull chicks and adults ringed with PVC ring during the period 2005-2014 in four breeding colonies in Gipuzkoa. The number of adults is shown in parenthesis.

Los pollos fueron marcados cuando tenían unos 20 días de edad (Cantos, 2000), entre los meses de junio y julio. En este programa se anilla sólo una fracción del número de pollos existentes en cada una de las colonias; esta fracción varió interanualmente según la disponibilidad de personal. Además, entre los meses de abril y mayo, se capturaron algunos ejemplares adultos con trampas de caída colocadas sobre el nido durante el periodo de incubación. Cada ejemplar se anilló con una anilla metálica y otra de PVC roja con código alfanumérico de cuatro dígitos en blanco.

Tras el abandono de las colonias, las aves son observadas por toda su área de distribución, normalmente en playas, rías, vertederos, lagunas, puertos y pueblos costeros. Estas observaciones se llevaron a cabo tanto por observadores del propio equipo de marcaje (Aranzadi) como por observadores que, a título voluntario, comunicaron sus avistamientos (Tabla 2).

Las observaciones de aves marcadas (en adelante, avistamientos) fueron registradas durante el periodo 01.08.2005-15.01.2015. La distribución geográfica de los avistamientos fue representada sobre cartografía de Europa mediante el programa QGIS con una proyección Mercator, que es la más recomendada para latitudes entre 750N y 7505. Los avistamientos se agruparon en un total de siete regiones: Cantábrico (provincias del Cantábrico así como Galicia y fachada atlántica francesa, desde Aquitania hasta el cabo Pern, en Finisterre), Portugal, Álava (dada la cercanía de esta provincia a las colonias de 


\begin{tabular}{|c|c|c|c|c|}
\hline \multirow{2}{*}{ Año } & \multicolumn{2}{|c|}{ Pollos } & \multicolumn{2}{|c|}{ Adultos } \\
\hline & Individuos & Avistamientos & Individuos & Avistamientos \\
\hline 2005 & 20 & 40 & 0 & 0 \\
\hline 2006 & 124 & 433 & 0 & 0 \\
\hline 2007 & 282 & 1225 & 6 & 14 \\
\hline 2008 & 392 & 1065 & 27 & 78 \\
\hline 2009 & 554 & 1640 & 43 & 111 \\
\hline 2010 & 576 & 1432 & 47 & 117 \\
\hline 2011 & 601 & 1393 & 31 & 66 \\
\hline 2012 & 645 & 1777 & 33 & 71 \\
\hline 2013 & 663 & 1792 & 26 & 87 \\
\hline 2014 & 538 & 1653 & 19 & 57 \\
\hline $2015^{*}$ & 66 & 81 & 0 & 0 \\
\hline Total & 4461 & 12531 & 232 & 601 \\
\hline
\end{tabular}

*Hasta el 15.01.2015

Tabla 2.- Número de individuos diferentes observados y total de avistamientos de gaviotas patiamarillas marcadas en cuatro colonias de Gipuzkoa, durante el periodo 2005-2015.

Table 2.-The number of individual yellow-legged gulls and total number of sightings of yellow-legged gulls ringed in four breeding colonies in Gipuzkoa, during the period 2005-2015.

origen, la presencia de individuos en el vertedero de la ciudad de Vitoria/Gasteiz podría ser debida a desplazamientos de forrajeo y no a una dispersión por la cuenca del río Ebro, motivo por el que esta provincia no se incluyó en la región "Ebro"), Mediterráneo (litoral de la fachada mediterránea, básicamente Cataluña y Comunidad Valenciana), Centro de España (observaciones en vertederos de Madrid y Castilla-La Mancha), Ebro (cuenca del río Ebro, excluido el litoral de la fachada mediterránea) y Reino Unido.

Durante el periodo de estudio se registraron un total de 13132 avistamientos, relativos a 1559 individuos diferentes (1500 marcados como pollos). Esto supone un 58,6\% de las aves que fueron marcadas. Por clases de edad, se avistaron un 57,7\% de las que se marcaron como pollos y un 93,7\% de las que lo fueron como adultos.

Un 98\% de los avistamientos tuvo lugar en el área cantábrica, incluida Galicia y Francia. El avistamiento de adultos se concentró, exclusivamente, en el área cantábrica (Tabla 3), por lo que posiblemente la dispersión de los adultos no exceda el ámbito del mar Cantábrico.

Casi la totalidad de los individuos que se marcaron como pollos y se avistó posteriormente se vio al menos una vez en el área cantábrica (99,34\%), independientemente de que, además, fuera visto en otras áreas. La presencia de aves marcadas en las zonas situadas fuera del ámbito cantábrico fue marginal: algo más del 1\% de los individuos fue avistado en 


\begin{tabular}{|l|rr|rr|}
\hline \multirow{2}{*}{ Región } & \multicolumn{2}{|c|}{ Pollos } & \multicolumn{2}{c|}{ Adultos } \\
& Individuos & Avistamientos & Individuos & Avistamientos \\
\hline \multirow{2}{*}{ Cantábrico } & $1490(99,34 \%)$ & $12310(98,24 \%)$ & $59(100 \%)$ & $601(100 \%)$ \\
Portugal & $17(1,12 \%)$ & $36(0,28 \%)$ & 0 & 0 \\
Álava & $12(0,80 \%)$ & $23(0,18 \%)$ & 0 & 0 \\
Mediterráneo & $7(0,46 \%)$ & $9(0,08 \%)$ & 0 & 0 \\
Centro España & $4(0,26 \%)$ & $150(1,20 \%)$ & 0 & 0 \\
Ebro & $2(0,12 \%)$ & $2(0,02 \%)$ & 0 & 0 \\
Reino Unido & $1(0,06 \%)$ & $1(0,01 \%)$ & 0 & 0 \\
\hline
\end{tabular}

Tabla 3.- Regiones de avistamiento de gaviotas patiamarillas marcadas en diferentes colonias en Gipuzkoa (Cantábrico oriental). Un mismo individuo puede ser visto en más de una región. El porcentaje de individuos y de avistamientos se ha calculado en relación al número global de individuos diferentes avistados o del total de avistamientos, respectivamente.

Table 3. - Sighting areas of gulls ringed in four breeding colonies in Gipuzkoa. A single bird may be seen in more than one area. The percentage of individuals and sightings has been calculated in relation to the global number of individuals or sightings, respectively.

Portugal y $<1 \%$, en cada una del resto de zonas en las que las gaviotas marcadas fueron observadas (Álava, Mediterráneo, centro de España, cuenca del río Ebro y Reino Unido) (Tabla 3).

Tan sólo dos aves (0,12\% de la población de aves marcadas) fueron observadas en diferentes puntos del Valle del Ebro, concretamente un ejemplar en el vertedero de Culebrete (Tudela; observadores: R. Rodríguez, D. Calleja, X. Remírez, J. Remírez) y otro en el de Huesca (observador: J. C. Albero; Fig. 2), ambos en enero de 2015. El ejemplar avistado en Tudela se anilló en la isla de Santa Clara y el otro en Ulia.

Hasta la publicación de este artículo, la presencia de gaviotas patiamarillas de origen cantábrico en la cuenca del río Ebro no estaba descrita. Asimismo, prácticamente se triplica el número de observaciones en el Mediterráneo respecto a lo anteriormente descrito (Arizaga et al., 2010). Trabajos precedentes habían especulado sobre las rutas de unión entre el Cantábrico y el Mediterráneo (Martínez-Abrain et al., 2002; Rodríguez \& Muntaner, 2004; Arizaga et al., 2009), pero no había ninguna evidencia concreta de que las aves pudieran seguir una u otra ruta.

Aunque durante el periodo 2005-2014 el número de aves marcadas en las colonias de Gipuzkoa ha sido alto (especialmente pollos), sólo un escasísimo porcentaje (0,12\%) ha sido avistado en la cuenca del río Ebro. La razón más obvia es que el número de ejemplares de origen cantábrico en dispersión hacia el Mediterráneo es bajo, y a que el tiempo de tránsito es probablemente corto. También contribuye el relativamente bajo esfuerzo de observación en comparación con otras zonas. Así, aun habiendo más individuos 


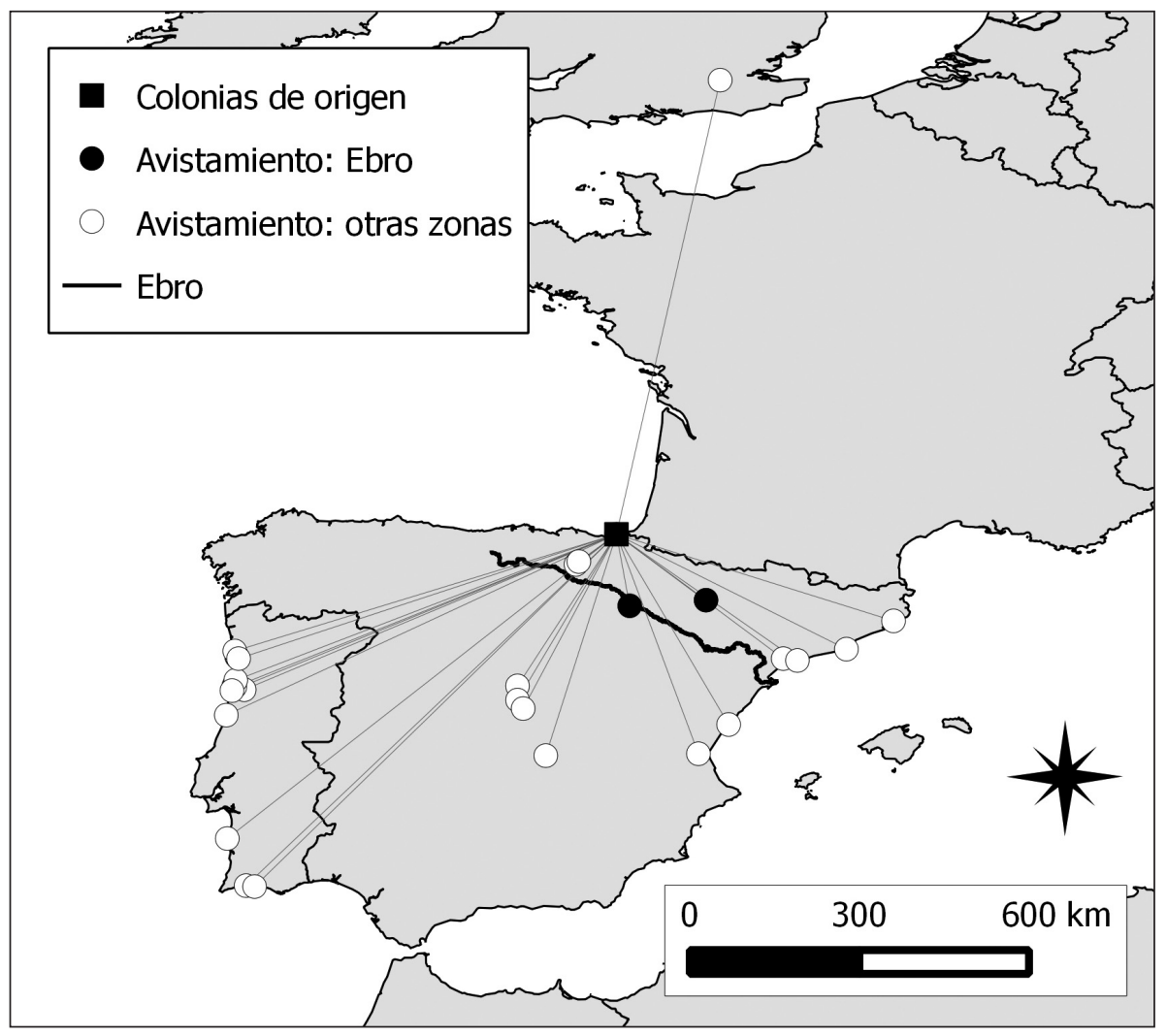

Fig. 2.- Localización de los sitios en los que se avistaron gaviotas patiamarillas marcadas en Gipuzkoa. No se incluyen en este mapa los avistamientos del área cantábrica, incluido el noroeste peninsular (Galicia).

Fig. 2.- Location of the sites where the sightings of yellow-legged gulls ringed in Gipuzkoa were obtained. Sighting data from the Bay of Biscay, including Galicia, have been removed from the map.

observados en las costas del mediterráneo que en el centro peninsular, son menos los individuos que se ven en la cuenca del Ebro de los que se observan en el centro de España. Sin duda, esto podría ser debido al gran esfuerzo de muestreo (G. Martín, com. pers.) que, durante los últimos años, se está llevando a cabo en, principalmente, determinados vertederos de Madrid. Allí se acumula el 1,2\% de las observaciones de aves marcadas como pollos en Gipuzkoa, si bien a esta zona sólo llegaría un 0,26\% de todos ellos.

La observación de gaviotas de origen cantábrico en dos puntos de la cuenca del Ebro, sumado a la ausencia de observaciones tanto al norte de los Pirineos como a lo largo de la costa del sur de España, daría cierto apoyo la idea de que el movimiento de gaviotas entre la costa cantábrica y el Mediterráneo sucede a través del Valle del Ebro. Para confirmar este hecho, haría falta, no obstante, más información. 


\section{Agradecimientos}

Un proyecto de esta naturaleza no hubiera sido posible sin la colaboración de cientos de voluntarios, a quienes damos nuestro más sincero agradecimiento. El proyecto ha sido parcialmente financiado por el Gobierno Vasco, la Diputación de Gipuzkoa y el Ayuntamiento de Donostia-S. Sebastián. E. Barba, A. Martínez-Abraín y A. Cama proporcionaron valiosos comentarios que ayudaron a mejorar una primera versión del trabajo.

\section{Bibliografía}

- Arizaga, J., Herrero, A., Aldalur, A. 2009. Primera cita en el Mediterráneo de un ejemplar de Gaviota Patiamarilla (Larus michahellis) nacido en el Cantábrico. Rev. Anillamiento 23: 6-9.

- Arizaga, J., Herrero, A., Galarza, A., Hidalgo, J., Aldalur, A., Cuadrado, J.F., Ocio, G. 2010. Firstyear movements of Yellow-legged Gull (Larus michahellis lusitanius) from the southeastern Bay of Biscay. Waterbirds 33: 444-450.

- Bermejo, A., Mouriño, J. 2003. Gaviota Patiamarilla, Larus cachinnans. En: Atlas de las aves reproductoras de España. Martí, R., Del Moral, J.C. (Ed.): 272-273. DGCN-SEO/BirdLife. Madrid.

- Cantos, F.J. 2000. El anillamiento científico en colonias de láridos. Rev. Anillamiento 6: 12-23.

- Geroudet, P. 1984. Origine mediterranéenne confirmée pour les Goélands leucophées du Léman. Nos oiseaux 37: 240.

- Martínez-Abrain, A., Oro, D., Carda, J., Del Señor, X. 2002. Movements of Yellow-Ledged Gulls Larus [cachinnans] michahellis from two small western Mediterranean colonies. Atlantic Seabirds 4: 101-108.

- Munilla, I. 1997. Desplazamientos de la Gaviota Patiamarilla (Larus cachinnans) en poblaciones del norte de la Península Ibérica. Ardeola 44: 19-26.

- Olsen, K. M. , Larson, H. 2004. Gulls of Europe, Asia and North America. Christopher Helm. London.

- Pons, J. M., Crochet, P. A., Thery, M. , Bermejo, A. 2004. Geographical variation in the yellowledged gull: introgression or convergence from the herring gull? J. Zool. Syst. Evol. Res. 42: $245-256$.

- Rodríguez, A., Muntaner, J. 2004. Primeros resultados del marcado de gaviota patiamarilla Larus michahellis con anillas de lectura en las Islas Baleares. A.O.B. 19: 69-77.

- Yésou, P. 1991. The sympatric breeding of Larus fuscus, L. cachinnans and L. argentatus in western France. Ibis 133: 256-263.

\footnotetext{
- Fecha de recepción/Date of reception: 07-04-2015

- Fecha de aceptación/Date of acceptance: 21-08-2015

Editor Asociado/Associate editor: Emilio Barba
} 